\title{
Role of dense matter in collective supernova neutrino transformations
}

\author{
A. Esteban-Pretel, ${ }^{1}$ A. Mirizzi, ${ }^{2}{ }^{3}$ S. Pastor, ${ }^{1}$ R. Tomàs, ${ }^{4}$ G. G. Raffelt,${ }^{2}$ P. D. Serpico, ${ }^{5}$ and G. Sigl ${ }^{4,6}$ \\ ${ }^{1}$ Institut de Física Corpuscular (CSIC-Universitat de València), \\ Edifici Instituts d'Investigació, Apt. 22085, 46071 València, Spain \\ ${ }^{2}$ Max-Planck-Institut für Physik (Werner-Heisenberg-Institut), Föhringer Ring 6, 80805 München, Germany \\ ${ }^{3}$ Istituto Nazionale di Fisica Nucleare, Roma, Italy \\ ${ }^{4}$ II. Institut für theoretische Physik, Universität Hamburg, Luruper Chaussee 149, 22761 Hamburg, Germany \\ ${ }^{5}$ Center for Particle Astrophysics, Fermi National Accelerator Laboratory, Batavia, IL 60510-0500, USA \\ ${ }^{6} \mathrm{APC}^{*}$ (AstroParticules et Cosmologie), 10, rue Alice Domon et Léonie Duquet, 75205 Paris Cedex 13, France
}

(Dated: 3 July 2008)

\begin{abstract}
For neutrinos streaming from a supernova $(\mathrm{SN})$ core, dense matter suppresses self-induced flavor transformations if the electron density $n_{e}$ significantly exceeds the neutrino density $n_{\nu}$ in the conversion region. If $n_{e}$ is comparable to $n_{\nu}$ one finds multi-angle decoherence, whereas the standard self-induced transformation behavior requires that in the transformation region $n_{\nu}$ is safely above $n_{e}$. This condition need not be satisfied in the early phase after SN core bounce. Our new multi-angle effect is a subtle consequence of neutrinos traveling on different trajectories when streaming from a source that is not point-like.
\end{abstract}

PACS numbers: 14.60.Pq, 97.60.Bw

\section{INTRODUCTION}

The neutrinos streaming from a supernova (SN) core or from the accretion torus of coalescing neutron stars are so dense that the neutrino-neutrino interaction causes collective flavor transformations 1, 2, 3, 4, 5, 6, 7, 8, 9, 10, 11, 12, 13, 14, 15, 16, 17, 18, 19, 20, 21, 22, 23, 24, $25,26,27,28]$. At the same time, the density of ordinary matter is also very large, suppressing normal flavor conversions unless the neutrinos encounter an MSW resonance [29, 30, 31, 32]. On the other hand, one of the many surprises of self-induced flavor transformations has been that dense matter barely affects them. They are driven by an instability in flavor space that is insensitive to matter because it affects all neutrino and antineutrino modes in the same way. Therefore, it can be transformed away by going to a rotating frame in flavor space [7, 8] in a sense to be explained below. Various numerical simulations confirm this insight.

We here clarify, however, that the matter density can not be arbitrarily large before it affects collective flavor conversions after all. The matter term is "achromatic" only if we consider the time-evolution of a homogeneous (but not necessarily isotropic) neutrino ensemble on a homogeneous and isotropic matter background. If the matter background is not isotropic, the current-current nature of the neutrino-electron interaction already implies that different neutrino modes experience a different matter effect.

It is more subtle that even without a current, matter still affects different neutrino modes differently if we study neutrinos streaming from a source. The relevant

\footnotetext{
*UMR 7164 (CNRS, Université Paris 7, CEA, Observatoire de Paris)
}

evolution is now the flavor variation of a stationary neutrino flux as a function of distance. For a spherically symmetric situation, "distance from the source" is uniquely given by the radial coordinate $r$. Neutrinos reaching a certain $r$ have travelled different distances on their trajectories if they were emitted with different angles relative to the radial direction. Therefore, at $r$ they have accrued different oscillation phases even if they have the same vacuum oscillation frequency and even if they have experienced the same matter background. In other words, if we project the flavor evolution of different angular modes on the radial direction, they have different effective vacuum oscillation frequencies even if they have the same energy. The same argument applies to matter that modifies the oscillation frequency in the same way along each trajectory, but therefore acts differently when expressed as an effective oscillation frequency along the radial direction.

The neutrino-neutrino interaction, when it is sufficiently strong, forces different modes to reach a certain $r$ with the same oscillation phase. To achieve this "selfmaintained coherence" the neutrino-neutrino term must overcome the phase dispersion that would otherwise occur. Such a dispersion is caused not only by a spectrum of energies, but also by a matter background.

To clarify and quantify these ideas we revisit in Sec. II the time evolution of a homogeneous ensemble and introduce the notion of the "rotation-averaged equations of motion." In Sec. III we discuss the geometric modifications when we study neutrinos streaming from a source and quantify the multi-angle effect caused by ordinary matter. We conclude in Sec. IV with a discussion of the implications of our findings for realistic situations relevant for supernova neutrinos. 


\section{HOMOGENEOUS ENSEMBLE}

\section{A. Isotropic background}

To understand the role of matter in collective neutrino transformations we begin with the equations of motion (EOMs) in their simplest form, relevant for a homogeneous (but not necessarily isotropic) gas of neutrinos. We only consider two-flavor oscillations where the most economical way to write the EOMs is in terms of the usual flavor polarization vectors $\mathbf{P}_{\mathbf{p}}$ for each mode $\mathbf{p}$ and analogous vectors $\overline{\mathbf{P}}_{\mathbf{p}}$ for the antineutrinos,

$$
\dot{\mathbf{P}}_{\mathbf{p}}=\mathbf{H}_{\mathbf{p}} \times \mathbf{P}_{\mathbf{p}}
$$

The "Hamiltonian" is

$$
\mathbf{H}_{\mathbf{p}}=\omega_{\mathbf{p}} \mathbf{B}+\lambda \mathbf{L}+\mu \int \mathrm{d} \mathbf{q}\left(\mathbf{P}_{\mathbf{q}}-\overline{\mathbf{P}}_{\mathbf{q}}\right)\left(1-\mathbf{v}_{\mathbf{q}} \cdot \mathbf{v}_{\mathbf{p}}\right)
$$

with $\mathrm{d} \mathbf{q}=\mathrm{d}^{3} \mathbf{q} /(2 \pi)^{3}$. We have used the vacuum oscillation frequency $\omega_{\mathbf{p}}=\Delta m^{2} / 2 E$ with $E=|\mathbf{p}|$ for relativistic neutrinos, $\mathbf{B}$ is a unit vector in the mass direction in flavor space, and $\mathbf{L}$ is a unit vector in the weak-interaction direction with $\mathbf{B} \cdot \mathbf{L}=\cos 2 \theta$ and $\theta$ the vacuum mixing angle. The effect of a homogeneous and isotropic medium is parametrized by $\lambda=$ $\sqrt{2} G_{\mathrm{F}}\left(n_{e^{-}}-n_{e^{+}}\right)$whereas the neutrino-neutrino term is given by $\mu=\sqrt{2} G_{\mathrm{F}} n_{\bar{\nu}_{e}}$. For simplicity we here assume that initially only $\nu_{e}$ and $\bar{\nu}_{e}$ are present with an excess neutrino density of $n_{\nu_{e}}=(1+\epsilon) n_{\bar{\nu}_{e}}$ [13]. The polarization vectors are initially normalized such that $\left|\int \mathrm{d} \mathbf{p} \overline{\mathbf{P}}_{\mathbf{p}}\right|=1$ and $\left|\int \mathrm{d} \mathbf{p} \mathbf{P}_{\mathbf{p}}\right|=1+\epsilon$. For antineutrinos the Hamiltonian is the same with $\omega_{\mathbf{p}} \rightarrow-\omega_{\mathbf{p}}$.

The matter term is "achromatic" in that it affects all modes of neutrinos and antineutrinos in the same way. It was first pointed out by Duan et al. [7] that therefore one may study the EOMs in a coordinate system that rotates around $\mathbf{L}$ with frequency $\lambda$ so that the matter term disappears. In the new frame the vector $\mathbf{B}$ rotates fast around $\mathbf{L}$ so that its transverse component averages to zero. Therefore, in the new frame the rotation-averaged Hamiltonian is

$$
\langle\mathbf{H}\rangle=\omega \cos (2 \theta) \mathbf{L}+\mu(\mathbf{P}-\overline{\mathbf{P}}),
$$

where for the moment we consider the even simpler case of an isotropic and monochromatic neutrino ensemble where the entire system is described by one polarization vector $\mathbf{P}$ for neutrinos and one $\overline{\mathbf{P}}$ for antineutrinos.

A dense matter background effectively projects the EOMs on the weak-interaction direction. In particular, the relevant vacuum oscillation frequency is now $\omega \cos 2 \theta$. For a small mixing angle, the case usually considered in this context, this projection effect is not important. However, a large mixing angle would strongly modify the projected $\omega$. Maximal mixing where $\cos 2 \theta=0$ would prevent any collective flavor transformations, an effect that is easily verified in numerical examples.
One usually assumes that the (anti)neutrinos are prepared in interaction eigenstates so that initially $\mathbf{P}$ and $\overline{\mathbf{P}}$ are oriented along $\mathbf{L}$. Therefore, the rotation-averaged EOMs alone do not lead to an evolution. However, in the unstable case of the inverted mass hierarchy, an infinitesimal disturbance is enough to excite the transformation. The fast-rotating transverse $\mathbf{B}$ component that was left out from the EOM is enough to trigger the evolution, but otherwise plays no crucial role [7, 9].

If we consider a homogeneous system where $\mu$ is a slowly decreasing function of time, one can find the adiabatic solution of the EOM for the simple system consisting only of $\mathbf{P}$ and $\overline{\mathbf{P}}$ [11, 15]. In vacuum, this is a complicated function of $\epsilon$ and $\cos 2 \theta$. In dense matter, however, we are effectively in the limit of a vanishing mixing angle because the initial orientation of the polarization vectors now coincides with the direction relevant for the rotation-averaged evolution. The original vacuum mixing angle only appears in the expression for the projected oscillation frequency $\omega \cos 2 \theta$.

With $z=\bar{P}_{z}$ the adiabatic connection between $\bar{P}_{z}$ and $\mu$ is now given by the inverse function of

$$
\frac{\omega \cos 2 \theta}{\mu}=\frac{\epsilon+2 z}{2}-\frac{\epsilon+2 z+(3 \epsilon+2 z) z}{2 \sqrt{(1+z)(1+z+2 \epsilon)}},
$$

where $-1 \leq z \leq+1$. The "synchronization radius" $r_{\text {sync }}$ where the adiabatic curve begins its decrease is implied by $\bar{P}_{z}=z=1$. One finds the familiar result $[9,[1]]^{1}$

$$
\left.\frac{\omega \cos 2 \theta}{\mu}\right|_{\text {sync }}=\frac{(\sqrt{1+\epsilon}-1)^{2}}{2} .
$$

For $\mu$ values larger than this limit, the polarization vectors are stuck to the $\mathbf{L}$ direction.

Without matter one finds that $(\mathbf{P}-\overline{\mathbf{P}}) \cdot \mathbf{B}$ is conserved [9]. Here, the analogous conservation law applies to $(\mathbf{P}-\overline{\mathbf{P}}) \cdot \mathbf{L}$. Therefore, the adiabatic solution for $P_{z}$ is such that $P_{z}-\bar{P}_{z}$ is conserved, i.e., $P_{z}=\bar{P}_{z}+\epsilon$.

In summary, the presence of dense matter simplifies the EOMs and in that the adiabatic solution is the one for a vanishing vacuum mixing angle, provided one uses the projected vacuum oscillation frequency.

\section{B. Background flux}

As a next example we still consider a homogeneous system, but now allow for a net flux of the background matter, assuming axial symmetry around the direction defined by the flux. For simplicity we consider a monochromatic ensemble with a single vacuum oscillation frequency $\omega$. We characterize the angular neutrino modes

\footnotetext{
${ }^{1}$ In these papers $\mu$ was normalized to the density of neutrinos and the results were expressed in terms of $\alpha=1 /(1+\epsilon)$. Here we have normalized $\mu$ to the density of antineutrinos and use the picture of an excess of neutrinos, expressed by $\epsilon$. For the same physical system our $\mu$ is the one of Ref. [9], divided by $1+\epsilon$.
} 
by their velocity component $v$ along the matter flux direction. The EOMs are in this case

$$
\dot{\mathbf{P}}_{v}=\mathbf{H}_{v} \times \mathbf{P}_{v} .
$$

The Hamiltonian for the mode $v$ is

$$
\mathbf{H}_{v}=\omega \mathbf{B}+\left(\lambda-\lambda^{\prime} v\right) \mathbf{L}+\mu(\mathbf{D}-v \mathbf{F}),
$$

where $\lambda^{\prime} \equiv \lambda v_{e}, v_{e}$ being the net electron velocity. Moreover,

$$
\begin{aligned}
& \mathbf{D}=\int_{-1}^{+1} \mathrm{~d} v\left(\mathbf{P}_{v}-\overline{\mathbf{P}}_{v}\right) \\
& \mathbf{F}=\int_{-1}^{+1} \mathrm{~d} v v\left(\mathbf{P}_{v}-\overline{\mathbf{P}}_{v}\right)
\end{aligned}
$$

are the net neutrino density and flux polarization vectors. The normalization is $\int \mathrm{d} v \overline{\mathbf{P}}_{v}=1$ and $\int \mathrm{d} v \mathbf{P}_{v}=1+\epsilon$.

Next, we transform the EOMs to a frame rotating with frequency $\lambda$, allowing us to remove the matter term, but not the matter flux,

$$
\left\langle\mathbf{H}_{v}\right\rangle=\left(\omega-\lambda^{\prime} v\right) \mathbf{L}+\mu(\mathbf{D}-v \mathbf{F}) .
$$

We assume $\theta$ to be small and thus use $\omega \approx \omega \cos 2 \theta$. We now have a system where the effective vacuum oscillation frequencies for neutrinos are uniformly distributed between $\omega \pm \lambda^{\prime}$ and for antineutrinos between $-\omega \pm \lambda^{\prime}$. Even after removing the average common precession of all modes, their evolution is still dominated by the matterflux term if $\lambda^{\prime} \gg \mu$. In other words, collective behavior now requires $\mu \gtrsim \lambda^{\prime}$ and not only $\mu \gtrsim \omega$.

The simplest example is the "flavor pendulum" where for $\epsilon=0$ and an isotropic neutrino gas one obtains the well-known pendular motions of the polarization vectors. Matter does not disturb this behavior, except that it takes logarithmically longer for the motion to start [9]. However, a matter flux, if sufficiently strong, suppresses this motion and the polarization vectors remain stuck to the $\mathbf{L}$ direction for both mass hierarchies. If the neutrino distribution is not isotropic, the ensemble quickly decoheres kinematically [12], an effect that is also suppressed by a sufficiently strong matter flux.

We have verified these predictions in several numerical examples, but have not explored systematically the transition between a "weak" and a "strong" matter flux because a homogeneous ensemble only serves as a conceptual example where matter can have a strong influence on self-induced transformations.

\section{SPHERICAL STREAM}

The most general case of neutrino flavor evolution consists of an ensemble evolving both in space and time. In practice, however, one usually considers quasi-stationary situations where one asks for the spatial flavor variation of a stationary neutrino flux streaming from a source.
The neutrino density decreases with distance so that one can mimic this situation by a homogeneous system evolving in time with a decreasing density, the expanding universe being a realistic example. However, the analogy has important limitations because collective oscillations introduce geometric complications into the spatialvariation case.

The simplest nontrivial example is a perfectly spherical source ("SN core") that emits neutrinos and antineutrinos like a blackbody surface into space. The matter background is also taken to be perfectly spherically symmetric, but of course varies with radius. As a further simplification we consider monochromatic neutrinos and antineutrinos that are all emitted with the same energy. Therefore, the only variable necessary to classify the neutrino modes is their angle of emission. The most useful variable is $u=\sin ^{2} \vartheta_{R}$ where $\vartheta_{R}$ is the angle of emission at the neutrino sphere at $r=R[13]$. At a distance $r$ the radial velocity of a mode $u$ is

$$
v_{u, r}=\sqrt{1-u(R / r)^{2}} .
$$

For blackbody-like emission the flux modes are uniformly distributed in the interval $0 \leq u \leq 1$.

The polarization vectors are taken to represent the neutrino flux density because it is the flux integrated over a sphere of radius $r$, not the density, that is conserved as a function of $r$. Ignoring a possible matter flux, the EOMs are 13]

$$
\partial_{r} \mathbf{P}_{u, r}=\mathbf{H}_{u, r} \times \mathbf{P}_{u, r},
$$

where the Hamiltonian is

$$
\mathbf{H}_{u, r}=\frac{\omega \mathbf{B}+\lambda_{r} \mathbf{L}}{v_{u, r}}+\mu_{r}\left(\frac{\mathbf{D}_{r}}{v_{u, r}}-\mathbf{F}_{r}\right) .
$$

For antineutrinos we have, as always, $\omega \rightarrow-\omega$. Since the polarization vectors describe the fluxes, the global density and flux polarization vectors are

$$
\begin{aligned}
& \mathbf{D}_{r}=\int_{0}^{1} \mathrm{~d} u \frac{\mathbf{P}_{u, r}-\overline{\mathbf{P}}_{u, r}}{v_{u, r}}, \\
& \mathbf{F}_{r}=\int_{0}^{1} \mathrm{~d} u\left(\mathbf{P}_{u, r}-\overline{\mathbf{P}}_{u, r}\right),
\end{aligned}
$$

using the normalization $\int_{0}^{1} \mathrm{~d} u \overline{\mathbf{P}}_{u, r}=1$ and $\int_{0}^{1} \mathrm{~d} u \mathbf{P}_{u, r}=$ $1+\epsilon$. The matter coefficient $\lambda_{r}=\sqrt{2} G_{\mathrm{F}}\left[n_{e^{-}}(r)-n_{e^{+}}(r)\right]$ encodes the effective electron density at radius $r$ whereas

$$
\mu_{r}=\mu_{R} \frac{R^{2}}{r^{2}} .
$$

Here, $\mu_{R}=\sqrt{2} G_{\mathrm{F}} \Phi_{\bar{\nu}_{e}}(R)$ with $\Phi_{\bar{\nu}_{e}}(R)$ the antineutrino flux at the neutrino sphere. Therefore, $\mu_{r}$ always varies as $r^{-2}$ due to the geometric flux dilution, whereas $\lambda_{r}$ is given by the detailed matter profile of a SN model 30 , 31, 32].

If we ignore for the moment the neutrino-neutrino term in Eq. (12), the EOM written in terms of the radial 
coordinate $r$ simply re-parametrizes the distance along the trajectory of a neutrino. Naturally, the oscillation pattern of a trajectory that is tilted relative to the radial direction produces a compressed oscillation pattern when projected on the radial direction. In the absence of neutrino-neutrino interactions, this simply means that along the radial direction the flavor variation of the global neutrino stream decoheres kinematically, even if the neutrinos are monochromatic and thus have the same vacuum oscillation frequency along their trajectories.

In the presence of neutrino-neutrino interactions, kinematical decoherence among different angular modes can still occur and in fact is a self-accelerating process if the asymmetry $\epsilon$ is too small. On the other hand, for a sufficient asymmetry, the radial variation of different angular modes is collective and they behave almost as if they were all emitted with the same angle relative to the radial direction. While this quasi single-angle behavior is theoretically not understood, numerically it has been consistently observed [8, 13, 18].

The variation of the polarization vectors with the common radial coordinate $r$ now acquires dynamical significance in that the polarization vectors evolve differently than they would in the absence of neutrino-neutrino interactions. From Eq. (12) it is obvious that the matter term is no longer the same for all modes and thus can not be transformed away by going to a rotating frame. This behavior does not depend on the radial variation of $\lambda_{r}$ - even a homogeneous medium would show this multiangle matter effect.

For quasi single-angle behavior to occur, $\epsilon$ must not be too small, a condition that is probably satisfied in a realistic SN. Therefore, the synchronization radius implied by Eq. (5) is always much larger than the neutrino-sphere radius $R$, allowing us to expand the EOMs in powers of $R / r \ll 1$. Using

$$
v_{u, r}^{-1}=1+\frac{u}{2} \frac{R^{2}}{r^{2}}
$$

we find

$$
\mathbf{H}_{u, r}=\left(\omega \mathbf{B}+\lambda_{r} \mathbf{L}\right)\left(1+\frac{u}{2} \frac{R^{2}}{r^{2}}\right)+\mu_{r} \frac{R^{2}}{2 r^{2}}\left(\mathbf{Q}_{r}+u \mathbf{F}_{r}\right)
$$

where

$$
\mathbf{Q}_{r}=\int_{0}^{1} \mathrm{~d} u u\left(\mathbf{P}_{u, r}-\overline{\mathbf{P}}_{u, r}\right)
$$

and $\mathbf{F}_{r}$ is the same as before. At large $r$ a small correction to $\omega$ is not crucial and can be ignored. The radial variation of $\lambda_{r}$ is slow compared to the precession, so we can go to a frame that rotates with a different frequency $\lambda_{r}$ at each radius. Finally the rotation-averaged Hamiltonian is

$$
\left\langle\mathbf{H}_{u, r}\right\rangle=\left(\omega+u \lambda_{r}^{*}\right) \mathbf{L}+\mu_{r}^{*}\left(\mathbf{Q}_{r}+u \mathbf{F}_{r}\right)
$$

where

$$
\begin{aligned}
\lambda_{r}^{*} & =\lambda_{r} \frac{R^{2}}{2 r^{2}}, \\
\mu_{r}^{*} & =\mu_{r} \frac{R^{2}}{2 r^{2}}=\mu_{R} \frac{R^{4}}{2 r^{4}}
\end{aligned}
$$

and we have assumed $\omega \cos 2 \theta \approx \omega$.

The multi-angle matter effect can be neglected if in the collective region beyond the synchronization radius we have

$$
\lambda_{r}^{*} \ll \mu_{r}^{*}
$$

equivalent to

$$
n_{e^{-}}-n_{e^{+}} \ll n_{\bar{\nu}_{e}} .
$$

In the opposite limit we expect that the large spread of effective oscillation frequencies prevents collective oscillations. In this case all polarization vectors remain pinned to the $\mathbf{L}$ direction and no flavor conversion occurs.

For intermediate values it is not obvious what will happen. One may expect that the multi-angle matter effect triggers multi-angle decoherence, destroying the quasi single-angle behavior. This indeed occurs for the inverted hierarchy whereas in the normal hierarchy we have not found any conditions where multi-angle decoherence was triggered by the multi-angle matter effect. We recall that for a sufficiently small $\epsilon$ multi-angle decoherence occurs even in the normal hierarchy whereas no collective transformation arise for a sufficiently large $\epsilon$ [13].

We illustrate these points with a numerical example where $R=10 \mathrm{~km}, \omega=0.3 \mathrm{~km}^{-1}, \theta=10^{-2}, \mu_{R}=$ $7 \times 10^{4} \mathrm{~km}^{-1}$ and

$$
\lambda_{r}=\lambda_{R}\left(\frac{R}{r}\right)^{n}
$$

with $n=2$. This particular value of the power-law index leads to the same radial dependence of $\mu_{r}^{*}$ and $\lambda_{r}^{*}$, see Eq. (19). In Fig. 1 we show the radial variation $\mu_{r}^{*}$ and $\lambda_{r}^{*}$ for different choices of $\lambda_{R}$, between $10^{3} \mathrm{~km}^{-1}$ and $10^{6} \mathrm{~km}^{-1}$. Even for the smallest matter effect, the ordinary MSW resonance, defined by the condition $\lambda_{r}=\omega$, stays safely beyond the collective region.

In Fig. 2 we show the corresponding variation of $\bar{P}_{z}$ for three different cases: inverted mass hierarchy and $\epsilon=$ 0.25 (top panel), inverted mass hierarchy and $\epsilon=0.06$ (middle panel), and normal mass hierarchy and $\epsilon=0.06$ (bottom panel). In the top panel we observe the usual transformation for a small matter effect, a complete suppression of transformations for a large matter effect, and multi-angle decoherence for intermediate cases. Repeating the same exercise for the normal mass hierarchy and the same $\epsilon$ reveals no macroscopic influence of the matter term.

For a sufficiently small $\epsilon$ one finds self-induced multiangle decoherence for both hierarchies. In the middle and bottom panels of Fig. 2 we show how with a sufficiently strong matter effect the decoherence can be suppressed for both hierarchies. 


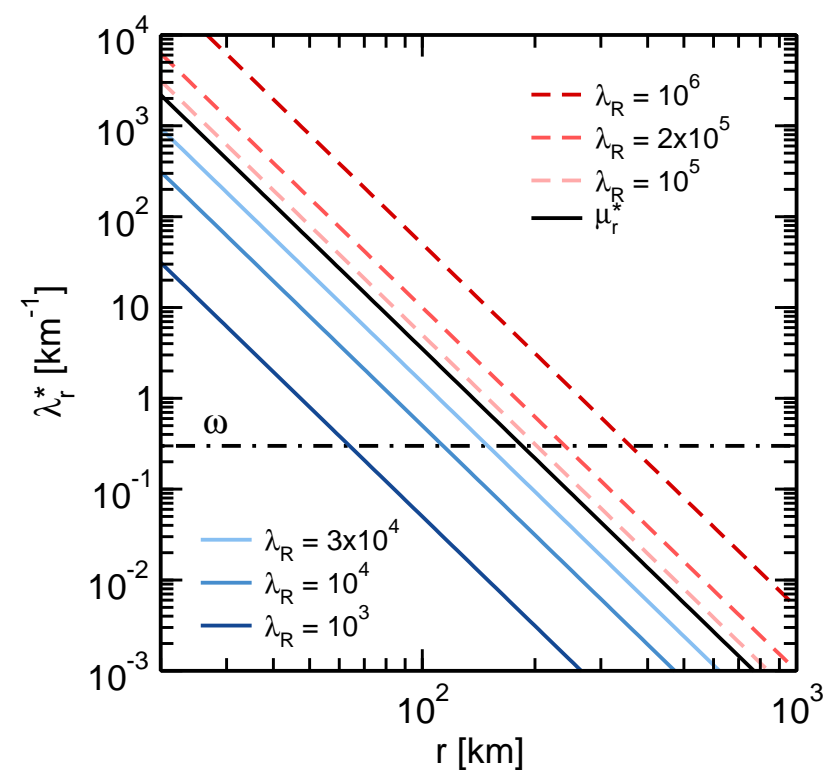

FIG. 1: Radial variation of $\mu_{r}^{*}$ and $\lambda_{r}^{*}$ for our numerical examples with the indicated value of $\lambda_{R}$. The value of $\omega$ considered in this work is also shown.

\section{DISCUSSION}

We have identified a new multi-angle effect in collective neutrino transformations that is caused by a matter background. Previous numerical studies of multi-angle effects had used a matter profile that satisfies the condition Eq. (21) in the critical region [8, 18]. In other multi-angle studies matter was entirely ignored [13] and otherwise, single-angle studies were performed. Therefore, the multi-angle matter effect discussed here had escaped numerical detection.

In many practical cases relevant for SN physics or in coalescing neutron stars, the density of matter is probably small enough so that this effect can be ignored. On the other hand, for iron-core SNe, during the accretion phase the matter density can reach values and can have such a profile that it is important. The possibility of such large matter densities had led some of us to speculate that the second-order difference between the $\nu_{\mu}$ and $\nu_{\tau}$ refractive effect could sometimes play an interesting role for three-flavor collective transformations [21]. It is easy to show, however, that the density requirement for this mu-tau effect to be important implies that the multiangle matter effect can not be avoided. In this sense, one complicated effect caused by a large matter density annihilates another one.

If at early times the matter density profile is such that our multi-angle effect is important, this will not be the case at later times when the explosion has occurred and the matter profile contracts toward the neutron star. In principle, therefore, interesting time-dependent features in the oscillation probability can occur.

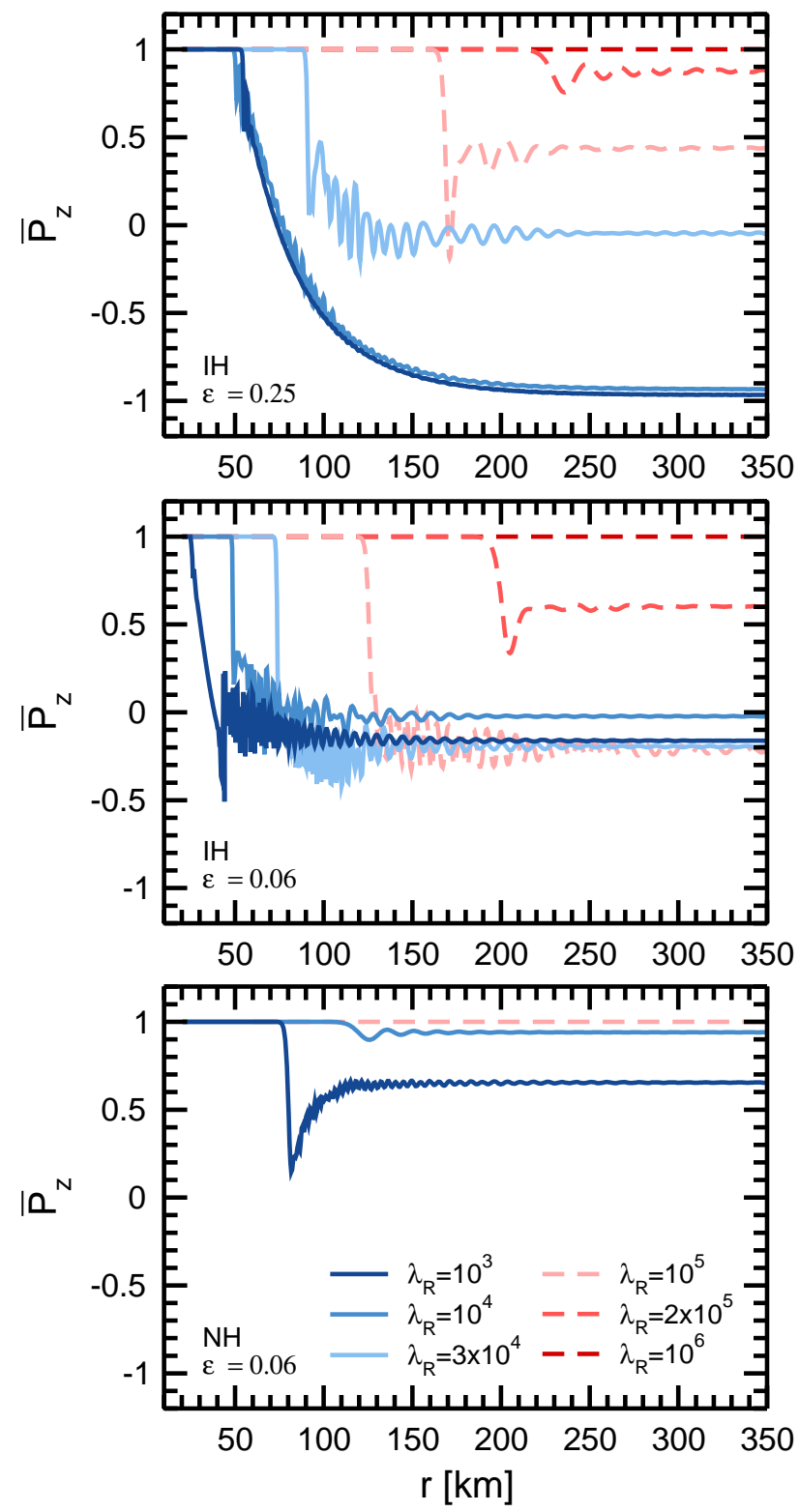

FIG. 2: Radial variation of $\bar{P}_{z}$ for three different scenarios: inverted mass hierarchy and $\epsilon=0.25$ (top panel), inverted mass hierarchy and $\epsilon=0.06$ (middle panel), and normal mass hierarchy and $\epsilon=0.06$ (bottom panel). In each panel different values of $\lambda_{R}$ have been assumed, reported in the bottom panel.

A large matter effect can be "rotated away" from the EOMs when it is identical for all modes. Here we have seen that even a perfectly uniform medium provides a multi-angle variation of the matter effect. We note that the matter fluxes would not be important, in contrast to our first example of a homogeneous ensemble, because the relevant quantity is the spread of the matter effect between different modes. Therefore, whenever a flux term would be important, the matter density term already provides a strong multi-angle effect. 
Possible polarizations of the electron-positron background in the strong SN magnetic fields would produce an additional axial term in the matter potential, proportional to the scalar product of the neutrino momentum with the electron polarization direction [33]. However, we expect that also this effect should be negligible with respect to the matter density term.

In addition, the medium can have density variations caused by convection and turbulence [34 that is known to affect the MSW resonance under certain circumstances [35, 36, 37, 38, 39]. Density variations in the transverse direction to the neutrino stream lines may well cause important variations of the matter effect between different modes. It remains to be investigated in which way collective flavor transformations are affected.

\section{Acknowledgments}

We acknowledge partial support by the DFG (Germany) under grants TR-27 and SFB-676, by The Cluster of Excellence "Origin and Structure of the Universe," by the European Union under the ILIAS project (contract No. RII3-CT-2004-506222) and an RT Network (contract No. MRTN-CT-2004-503369), and by the Spanish grant FPA2005-01269. A.E. is supported by a FPU grant from the Spanish Government, A.M. by INFN (Italy) through a post-doctoral fellowship, and P.S. by the US Department of Energy and by NASA grant NAG5-10842. Fermilab is operated by Fermi Research Alliance, LLC under Contract No. DE-AC02-07CH11359 with the United States Department of Energy.
[1] J.T. Pantaleone, Phys. Lett. B 287, 128 (1992).

[2] S. Samuel, Phys. Rev. D 48, 1462 (1993).

[3] Y.Z. Qian and G.M. Fuller, Phys. Rev. D 52, 656 (1995) arXiv:astro-ph/9502080.

[4] S. Pastor and G. Raffelt, Phys. Rev. Lett. 89, 191101 (2002) arXiv:astro-ph/0207281.

[5] R.F. Sawyer, Phys. Rev. D 72, 045003 (2005) arXiv:hep-ph/0503013.

[6] G.M. Fuller and Y.Z. Qian, Phys. Rev. D 73, 023004 (2006) arXiv:astro-ph/0505240.

[7] H. Duan, G.M. Fuller, and Y.Z. Qian, Phys. Rev. D 74, 123004 (2006) arXiv:astro-ph/0511275.

[8] H. Duan, G.M. Fuller, J. Carlson, and Y.Z. Qian, Phys. Rev. D 74, 105014 (2006) arXiv:astro-ph/0606616.

[9] S. Hannestad, G.G. Raffelt, G. Sigl, and Y.Y.Y. Wong, Phys. Rev. D 74, 105010 (2006); 76, 029901(E) (2007) arXiv:astro-ph/0608695.

[10] A.B. Balantekin and Y. Pehlivan, J. Phys. G 34, 47 (2007) arXiv:astro-ph/0607527.

[11] H. Duan, G.M. Fuller, J. Carlson, and Y.Z. Qian, Phys. Rev. D 75, 125005 (2007) arXiv:astro-ph/0703776.

[12] G.G. Raffelt and G. Sigl, Phys. Rev. D 75, 083002 (2007) arXiv:hep-ph/0701182

[13] A. Esteban-Pretel, S. Pastor, R. Tomàs, G.G. Raffelt, and G. Sigl, Phys. Rev. D 76, 125018 (2007) arXiv:0706.2498.

[14] G.G. Raffelt and A.Yu. Smirnov, Phys. Rev. D 76, 081301 (2007) arXiv:0705.1830.

[15] G.G. Raffelt and A.Yu. Smirnov, Phys. Rev. D 76, 125008 (2007) arXiv:0709.4641.

[16] H. Duan, G.M. Fuller, and Y.Z. Qian, Phys. Rev. D 76, 085013 (2007) arXiv:0706.4293.

[17] H. Duan, G.M. Fuller, J. Carlson, and Y.Z. Qian, Phys. Rev. Lett. 99, 241802 (2007) arXiv:0707.0290].

[18] G.L. Fogli, E. Lisi, A. Marrone, and A. Mirizzi, J. Cosmol. Astropart. Phys. 0712, 010 (2007)
arXiv:0707.1998.

[19] H. Duan, G.M. Fuller, J. Carlson, and Y.Z. Qian, Phys. Rev. Lett. 100, 021101 (2008) arXiv:0710.1271.

[20] B. Dasgupta, A. Dighe, A. Mirizzi, and G.G. Raffelt, Phys. Rev. D 77, 113007 (2008) arXiv:0801.1660.

[21] A. Esteban-Pretel, S. Pastor, R. Tomàs, G.G. Raffelt, and G. Sigl, Phys. Rev. D 77, 065024 (2008) arXiv:0712.1137.

[22] B. Dasgupta and A. Dighe Phys. Rev. D 77, 113002 (2008) arXiv:0712.3798.

[23] H. Duan, G.M. Fuller, and Y.Z. Qian, Phys. Rev. D 77, 085016 (2008) arXiv:0801.1363.

[24] B. Dasgupta, A. Dighe, and A. Mirizzi, Phys. Rev. Lett. (2008), in press arXiv:0802.1481.

[25] R.F. Sawyer, preprint arXiv:0803.4319

[26] H. Duan, G.M. Fuller, and J. Carlson, preprint arXiv:0803.3650

[27] S. Chakraborty, S. Choubey, B. Dasgupta, and K. Kar, J. Cosmol. Astropart. Phys. 0809, 013 (2008) [arXiv arXiv:0805.3131].

[28] B. Dasgupta, A. Dighe, A. Mirizzi, and G.G. Raffelt, Phys. Rev. D 78, 033014 (2008) arXiv:0805.3300.

[29] A.S. Dighe and A.Yu. Smirnov, Phys. Rev. D 62, 033007 (2000) arXiv:hep-ph/9907423.

[30] R.C. Schirato and G.M. Fuller, preprint arXiv:astro-ph/0205390

[31] G.L. Fogli, E. Lisi, D. Montanino, and A. Mirizzi, Phys. Rev. D 68, 033005 (2003) arXiv:hep-ph/0304056.

[32] R. Tomàs, M. Kachelriess, G. Raffelt, A. Dighe, H.T. Janka, and L. Scheck, J. Cosmol. Astropart. Phys. 0409, 015 (2004) arXiv:astro-ph/0407132.

[33] H. Nunokawa, V.B. Semikoz, A.Yu. Smirnov, and J.W.F. Valle, Nucl. Phys. B 501, 17 (1997) arXiv:hep-ph/9701420.

[34] L. Scheck, H.T. Janka, T. Foglizzo, and K. Kifonidis, Astron. Astrophys. 477, 931 (2008) arXiv:0704.3001. 
[35] F.N. Loreti, Y.Z. Qian, G.M. Fuller, and A.B. Balantekin, Phys. Rev. D 52, 6664 (1995) arXiv:astro-ph/9508106.

[36] G.L. Fogli, E. Lisi, A. Mirizzi, and D. Montanino, J. Cosmol. Astropart. Phys. 0606, 012 (2006) arXiv:hep-ph/0603033.

[37] A. Friedland and A. Gruzinov, preprint
arXiv:astro-ph/0607244

[38] S. Choubey, N.P. Harries, and G.G. Ross, Phys. Rev. D 76, 073013 (2007) arXiv:hep-ph/0703092.

[39] J.P. Kneller, G.C. McLaughlin, and J. Brockman, Phys. Rev. D 77, 045023 (2008) arXiv:0705.3835. 